\title{
High-Energy Limit of QCD beyond Sudakov Approximation
}

\author{
Tao $\mathrm{Liu}^{1}$ and Alexander A. Penin ${ }^{1,2}$ \\ ${ }^{1}$ Department of Physics, University of Alberta, Edmonton, Alberta T6G 2J1, Canadd * \\ ${ }^{2}$ Institut für Theoretische Teilchenphysik, Karlsruher Institut für Technologie (KIT), 76128 Karlsruhe, Germany
}

\begin{abstract}
We study the high-energy limit of the scattering amplitudes suppressed by the leading power of the quark mass in perturbative quantum chromodynamics. We prove the factorization and perform all-order resummation of the double-logarithmic radiative corrections which determine the asymptotic behavior of the amplitudes. In contrast to the Sudakov logarithms, the mass-suppressed double-logarithmic corrections are induced by soft quark exchange. The structure of the corrections and the asymptotic behavior of the amplitudes in this case crucially depend on the color flow in a given process and are determined by the eikonal color charge nonconservation. We present explicit results for the Higgs boson production in gluon fusion mediated by a light-quark loop and for the leading power-suppressed contributions to the quark form factors, which reveal "magical" universality. Nontrivial relations between the asymptotic behavior of different amplitudes and the amplitudes in different gauge theories are found.
\end{abstract}

PACS numbers: 11.15.Bt, 12.38.Bx, 12.38.Cy

Evaluating the scattering amplitudes in the highenergy limit is a fundamental problem of quantum field theory which remains in the focus of theoretical research for decades since the leading asymptotic behavior of an electron scattering amplitude in quantum electrodynamics (QED) has been derived in Ref. [1]. This behavior is determined by the "Sudakov" radiative corrections, which include the second power of the large logarithm of the electron mass divided by a characteristic momentum transfer of the process per each power of the fine structure constant. Sudakov logarithms exponentiate and result in a strong universal suppression of the scattering amplitudes in the limit when all the kinematic invariants of the process are large. Within different approaches the analysis has been extended to nonabelian gauge theories and to subleading logarithms [2 [6] , which is crucial for a wide class of applications from deep inelastic scattering to Drell-Yan processes and the Higgs boson production. This analysis however does not extend to the part of the amplitudes which is power suppressed in the high energy limit. The power-suppressed contributions now attract a lot of attention in various contexts (see e.g. $[7-18])$. Incorporating the logarithmically enhanced power-suppressed terms can significantly increase the accuracy and extend the region where the leading-power approximation is applicable. It becomes crucial when a power-suppressed term gives the leading contribution to a physical observable as in the case of the bottom-quark mediated Higgs boson production in gluon fusion. The latter is formally suppressed by the ratio $m_{b}^{2} / m_{H}^{2}$ of the quark mass $m_{b}$ to the Higgs boson mass $m_{H}$ but significantly changes the shape of the Higgs boson transverse momentum distribution. The effective expansion parameter in this case is $\ln ^{2}\left(m_{b}^{2} / m_{H}^{2}\right) \alpha_{s} \approx 40 \alpha_{s}$ rather than the strong coupling constant $\alpha_{s}$, and the resummation of the double-logarithmic corrections is mandatory for a reliable theoretical prediction [8, 13]. In general very little is known so far about the all-order structure of such corrections. In contrast to Sudakov logarithms they do not exponentiate and do not factorize into the wave functions of scattering particle. A few known examples of the all-order resummation are restricted to abelian gauge theory [12, 13, 19, 20]. Extension of the analysis to quantum chromodynamics (QCD) is not straightforward and requires a systematic treatment of the factorization. In this Letter we present the factorization formula and perform the resummation of the double-logarithmic corrections to the amplitudes suppressed by the leading power of quark mass. The Letter is organized as follows. First we briefly describe the idea of the method with a simple example which retains all the features of the general problem. Then we apply the resulting factorization formula to derive the double-logarithmic approximation for the amplitude of the Higgs boson production in gluon fusion mediated by a light-quark loop and for the leading power-suppressed contribution to the quark scattering in the external vector, axial, scalar and pseudoscalar fields. Finally we summarize the qualitative features and discuss universality of our solution for different amplitudes and gauge models.

To introduce the main idea of our approach we consider an amplitude $\mathcal{G}$ for the scattering of a quark of mass $m_{q}$, initial momentum $p_{1}$ and final momentum $p_{2}$, by a local operator $\left(G_{\mu \nu}^{a}\right)^{2}$ of the gauge field strength tensor. The origin of such a vertex is not relevant for our discussion and one may suggest that it describes the gluon field interaction to the Higgs boson mediated by an infinitely heavy quark loop. We consider the limit of the on-shell quark $p_{1}^{2}=p_{2}^{2}=m_{q}^{2}$ and the large Euclidean momentum transfer $Q^{2}=-\left(p_{2}-p_{1}\right)^{2}$ when the ratio $\rho \equiv m_{q}^{2} / Q^{2}$ is positive and small. In the light-cone coordinates $p_{1} \approx p_{1}^{-}$and $p_{2} \approx p_{2}^{+}$. The leading-order scattering is given by the one-loop diagram in Fig. 1(a). Conservation of helicity at high energy requires a helicity 


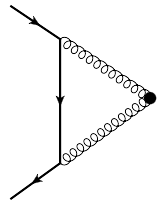

(a)

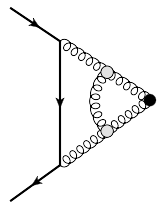

(b)

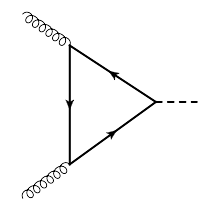

(c)

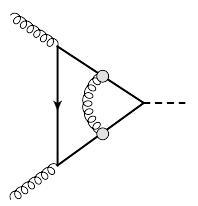

(d)

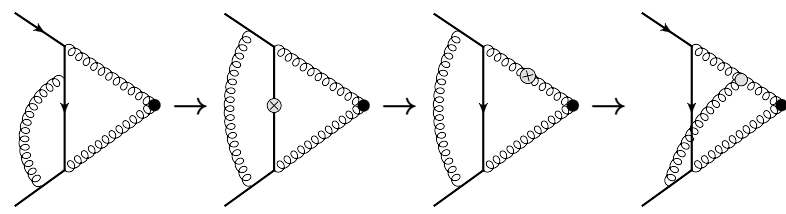

(a) (c)
FIG. 1. The leading order one-loop Feynman diagrams for (a) quark scattering by the $\left(G_{\mu \nu}^{a}\right)^{2}$ vertex (black circle) and (c) the Higgs boson production in gluon fusion. The diagrams (b) and (d) with the effective vertices (gray circles) defined in the text represent the non-Sudakov double-logarithmic corrections to the process (a) and (c), respectively.

flip on the virtual quark line. As a consequence at high energy the amplitude is suppressed by the first power of $m_{q}$. The virtual quark propagator then can be approximated as follows $S(l) \approx \frac{m_{q}}{l^{2}-m_{q}^{2}}$. For $m_{q} \ll l \ll Q$ the gauge boson propagators are eikonal i.e. proportional to $\frac{1}{2 p_{i} l}$, and the diagram has the double-logarithmic scaling. Thus we have a typical situation when a soft quark exchange generates the double-logarithmic contribution to the mass-suppressed amplitude. As we see, the emission of the soft quark results in the change of the color group representation of a particle propagating along the eikonal line, or the eikonal color charge nonconservation. This is a crucial feature of the process which plays an important role in further analysis. Let us now consider the radiative corrections to the amplitude and, for a moment, focus on an abelian case of the photon interaction. Then in a covariant gauge the double-logarithmic corrections are produced by a soft photon exchange between the external quark lines or an external and the virtual quark lines. The key idea of the approach is to move the soft photon vertex from the virtual soft quark line to an eikonal photon line through a sequence of identities graphically represented in Fig. 2, Let us describe this sequence in more detail. In a covariant gauge only $A^{-}$ light-cone component of the photon field can be emitted by the eikonal quark line with the momentum $p_{2}$, while the emission of the $A^{+}$and transverse components is suppressed. Thus we can use the Ward identity to convert the diagram Fig. 2(a) into Fig. 2(b) where the crossed circle on the quark propagator correspond to the replacement $S(l) \rightarrow S(l)-S\left(l+l_{g}^{+}\right)$, with $l_{g}$ being the soft photon momentum. By the momentum shift $l \rightarrow l-l_{g}^{+}$ in the second term of the above expression the crossed circle can be moved to the eikonal line which becomes $\frac{1}{2 p_{1} l}-\frac{1}{2 p_{1}\left(l+l_{g}^{+}\right)}$, Fig. 2(c). The opposite eikonal line is not sensitive to this shift since $p_{2}^{-} \approx 0$. Finally by using the inverted identity we transform the diagram Fig. 2(c) into Fig. 2(d) with an effective dipole coupling $2 e_{q} p_{1}^{\mu}$ to the photon line, where $e_{q}$ is the quark charge. Since $p_{1}^{+} \approx 0$ we can replace $p_{1} l_{g}^{+}$by $p_{1} l_{g}$ in the gauge boson propagator as long as $l_{g} \ll Q$ and after adding the sym-
FIG. 2. Diagramatic representation of the sequence of identities which move the soft gauge boson vertex from the soft quark to the eikonal gauge boson line, as explained in the text.

metric diagram we get a structure characteristic to the standard eikonal factorization picture. This factorization, however, requires the summation over all possible insertions of the soft photon vertex along each eikonal line while in the case under consideration the diagram in Fig. 1(b) with the soft exchange between the photon lines is missing. This diagram can be added to complete the factorization and then subtracted. Thus after factoring out the soft photon exchange between the external quark lines the remaining soft photon contribution is given by the diagram Fig. 1(b) with the coefficient $-e_{q}^{2}$. Note that the first Ward identity of the sequence in Fig. 1 is sufficient to prove the factorization of the soft photons with the momentum $l_{g} \ll m_{q}$ as it has been done in the original paper [21]. This algorithm however does not work for the momentum interval $m_{q} \ll l_{g} \ll Q$ which does contribute to the double-logarithmic corrections. Our method extends the factorization to this region at the expense of introducing the above subtraction term, which compensates the charge variation of the eikonal line after the soft quark emission.

The above result can be generalized to QCD in a straightforward way. The difference with respect to the abelian case is that $e_{q}^{2}$ should be replaced by the quadratic Casimir operator of the fundamental representation $C_{F}$ and the contribution similar to Fig. 1(b) does exist in QCD due to gluon self-coupling and is proportional to the quadratic Casimir operator the adjoint representation $C_{A}$. Thus the part of the soft gluon exchange which does not factorize into external lines is given by the diagram Fig. 1(b) with the color weight $C_{A}-C_{F}$, which directly links it to the variation of the color charge along the eikonal lines. We have verified the above factorization by explicit evaluation of the two-loop corrections in the high-energy limit within the expansion by regions framework 22 24]. Since the emission of the soft gluons from an eikonal line of a given color charge factorizes and exponentiates [25] we can apply the above trick to an arbitrary number of soft gluons. Hence the factorization formula for the double-logarithmic corrections to the amplitude becomes

$$
\mathcal{G}=Z_{q}^{2} g(-z) \mathcal{G}^{(0)},
$$


where $\mathcal{G}^{(0)}$ is the leading-order one-loop amplitude, $Z_{q}^{2}$ is the standard Sudakov factor for a quark scattering, and the function $g(-z)$ incorporates the non-Sudakov contribution of Fig. 1(b) with an arbitrary number of the effective soft gluon exchanges. The Sudakov factor reads

$$
Z_{q}^{2}=\exp \left[-C_{F}\left(\frac{\alpha_{s}}{2 \pi} \frac{\ln \rho}{\varepsilon}+x\right)\right]
$$

where $x=\frac{\alpha_{s}}{4 \pi} \ln ^{2} \rho$ is the double-logarithmic variable and dimensional regularization with $d=4-2 \varepsilon$ is used for the infrared divergences. The function $g(z)$ of the variable $z=\left(C_{A}-C_{F}\right) x$ is normalized to $g(0)=1$ and can be obtained by the standard method [12, 13, 18] in the form of the two-fold integral

$$
g(z)=2 \int_{0}^{1} \mathrm{~d} \xi \int_{0}^{1-\xi} \mathrm{d} \eta e^{2 z \eta \xi}
$$

over the normalized logarithmic variables $\eta=\ln v / \ln \rho$, $\xi=\ln u / \ln \rho$ related to the Sudakov parametrization of the soft quark momentum $l=u p_{1}+v p_{2}+l_{\perp}$. The argument of the exponent in Eq. (3) corresponds to the single soft gluon contribution. The integral Eq. (3) can be solved in terms of the generalized hypergeometric function

$$
g(z)={ }_{2} F_{2}(1,1 ; 3 / 2,2 ; z / 2)=2 \sum_{0}^{\infty} \frac{n !}{(2 n+2) !}(2 z)^{n}
$$

with the following asymptotic behavior at $z \rightarrow \infty$

$$
g(-z) \sim \frac{\ln (2 z)+\gamma_{E}}{z}, \quad g(z) \sim\left(\frac{2 \pi e^{z}}{z^{3}}\right)^{1 / 2}
$$

where $\gamma_{E}=0.577215 \ldots$ is the Euler constant. We have confirmed the perturbative expansion of Eq. (1) to $\mathcal{O}\left(\alpha_{s}^{3}\right)$ by explicit evaluation of the three-loop doublelogarithmic term adopting the method of Ref. [18].

The above equations determine the amplitude $\mathcal{G}$ in the high-energy limit in double-logarithmic approximation. Though this amplitude is of no particular phenomenological interest, the result can be used to find the solution for the amplitude of Higgs boson production in gluon fusion mediated by a bottom-quark loop mentioned in the introduction. Indeed, in the leading order this amplitude is given by the diagram in Fig. 1(c). Since the eikonal lines are characterized by the momentum and color charge but not spin, the only difference with respect to the previous case is the direction of the color flow. Hence the diagram in Fig. 1(d) which incorporates the non-Sudakov part of the correction corresponds to the same function $g(z)$ with the opposite sign of the argument and the factorization formula takes the form

$$
\mathcal{M}_{g g \rightarrow H}^{b}=-Z_{g}^{2} g(z)\left(\frac{3}{2} \ln ^{2} \rho \rho\right) \mathcal{M}_{g g \rightarrow H}^{(0)},
$$

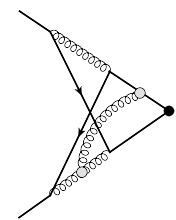

(a)

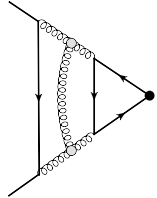

(b)

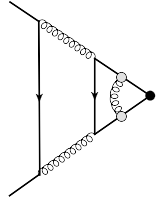

(c)
FIG. 3. The diagrams with an effective soft gluon exchange which incorporate the non-Sudakov double-logarithmic corrections to (a) vector and $(b, c)$ scalar form factor of a quark. The symmetric diagrams are not shown.

where $\rho=m_{b}^{2} / m_{h}^{2}$ is now a Minkowskian parameter, the heavy top-quark loop mediated amplitude is used as the leading order approximation $\mathcal{M}_{g g \rightarrow H}^{(0)}$ to have the mass suppression factor explicitly, and

$$
Z_{g}^{2}=\exp \left[-\frac{C_{A}}{\varepsilon^{2}} \frac{\alpha_{s}}{2 \pi}\right]
$$

is the Sudakov factor for a gluon scattering. We have verified Eq. (6) and, in particular, the relation between the diagrams imposed by the Ward identities by explicit twoloop calculation. The total two-loop contribution also agrees with the analytical result for the amplitude with an arbitrary value of the quark mass [26] expanded in the series in $\rho$.

Let us now consider a more complex problem of finding the asymptotic behavior of the leading mass-suppressed contribution to the amplitude of quark scattering in an external field. We start with the vector field case. At high energy and in the double-logarithmic approximation the deviation of the corresponding amplitude from the Born approximation is described by the Dirac form-factor $F_{1}$. Its asymptotic expansion can be written as follows

$$
F_{1}=Z_{q}^{2} \sum_{n} \rho^{n} F_{1}^{(n)},
$$

where $F_{1}^{(n)}$ are given by the power series in $\alpha_{s}$ with the coefficients depending on $\rho$ only logarithmically. Since the Sudakov corrections in Eq. (8) are factored out, in the double-logarithmic approximation the leading term of the expansion is just $F_{1}^{(0)}=1$. The double-logarithmic corrections to the leading power-suppressed term $F_{1}^{(1)}$ are induced by the nonplanar soft quark pair exchange and start with the two-loop contribution [12, 14, 18]. Following the algorithm described above we reduce the non-Sudakov part of the corrections to the diagram in Fig. 3(a), and the symmetric one. In these diagrams, as in Fig. 1(b), the effective soft gluon exchange with the color weight $C_{A}-C_{F}$ connects the eikonal lines with a given soft quark momentum. Note that in the double-logarithmic region the light-cone components of the soft quark momenta are ordered along the eikonal 


\begin{tabular}{c|c|c|c|c|c|c|c}
\hline \hline$n$ & 1 & 2 & 3 & 4 & 5 & 6 & 7 \\
\hline \hline $2^{n} n^{2} n ! c_{n}$ & $\frac{2}{5}$ & $\frac{88}{105}$ & $\frac{8}{7}$ & $\frac{70144}{51975}$ & $\frac{640}{429}$ & $\frac{25344}{15925}$ & $\frac{2727424}{1640925}$ \\
\hline \hline
\end{tabular}

TABLE I. The normalized coefficients of the Taylor series for the function $f(z)$, Eq. (10), up to $n=7$.

lines so that in each eikonal propagator only one momentum should be retained, which determines the position of the effective vertices in Fig. 3(a). The corresponding factorization formula for the leading power-suppressed term reads

$$
F_{1}^{(1)}=\frac{C_{F}\left(C_{A}-2 C_{F}\right)}{6} x^{2} f(-z),
$$

where the function $f(-z)$ incorporates the non-Sudakov contribution of Fig. [3(a) with an arbitrary number of the effective soft gluon exchanges and is normalized to the two-loop result $f(0)=1$. This function has an integral representation similar to Eq. (3)

$$
\begin{aligned}
f(z)= & 12 \int_{0}^{1} \mathrm{~d} \eta_{1} \int_{\eta_{1}}^{1} \mathrm{~d} \eta_{2} \int_{0}^{1-\eta_{2}} \mathrm{~d} \xi_{2} \int_{\xi_{2}}^{1-\eta_{1}} \mathrm{~d} \xi_{1} e^{2 z \eta_{1}\left(\xi_{1}-\xi_{2}\right)} \\
& \times e^{2 z \xi_{2}\left(\eta_{2}-\eta_{1}\right)}
\end{aligned}
$$

where the integration is performed over the logarithmic Sudakov variables for each soft quark momenta and the exponential factors correspond to Fig. 3(a) and the symmetric diagram. We are not able to solve the four-fold integral Eq. (10) in a closed analytic form. However, the coefficients of the series $f(z)=1+\sum_{n=1}^{\infty} c_{n} z^{n}$ can be computed for any given $n$ corresponding to the $(n+2)$ loop double-logarithmic contribution and have the following large- $n$ behavior $c_{n} \sim \frac{\ln n}{n ! 2^{n} n^{5 / 2}}$. The first seven coefficients of the series are listed in Table 【 The asymptotic behavior of the function at $z \rightarrow \infty$ reads

$$
f(-z) \sim C_{-}\left(\frac{\ln z}{z}\right)^{2}, \quad f(z) \sim C_{+} \ln z\left(\frac{e^{z}}{z^{5}}\right)^{1 / 2},
$$

where the constant $C_{-}=3.6 \ldots, C_{+}=14.8 \ldots$ are found numerically.

Let us now consider quark scattering in the external scalar field parametrized by the scalar form factor $F_{S}$. In the equivalent notations for the leading power-suppressed term $F_{S}^{(1)}$ we obtain

$$
F_{S}^{(1)}=-\frac{C_{F} T_{F}}{3} x^{2} f_{S}(-z),
$$

where $T_{F}=1 / 2$ and the function

$$
f_{S}(z)=24 \int_{0}^{1} \mathrm{~d} \eta_{1} \int_{0}^{1-\eta_{1}} \mathrm{~d} \xi_{1} \int_{\eta_{1}}^{1-\xi_{1}} \mathrm{~d} \eta_{2} \int_{\xi_{1}}^{1-\eta_{2}} \mathrm{~d} \xi_{2} e^{2 z \eta_{2} \xi_{2}} e^{-2 z \eta_{1} \xi_{1}}
$$

is determined by the planar diagrams in Fig. 3(b,c) with the corresponding exponential factors given separately. Amazingly, though the topology of the diagrams in Fig. 31(a) and Fig. 3(b,c) is completely different, Eqs. (10) and (13) describe the same function $f_{S}(z) \equiv f(z)$ as it can be easily verified. For the moment we do not have a plausible explanation of this universality. At the same time it is straightforward to extend the analysis to the axial $F_{A}$ and the pseudoscalar $F_{P}$ form factors, for which we obtain the result in the form of Eq. (91) and Eq. (12) with $f_{A}(z)=-f(z)$ and $f_{P}(z)=f(z)$, respectively.

Our results agree with the asymptotic expansion of the exact two-loop expressions for the form factors [2729]. The result for the vector form factor agrees with the explicit calculation of the three-loop double-logarithmic term [18] including the relations between the diagrams imposed by the Ward identities. For $C_{A}=0$ and $C_{F}=1$ Eq. (9) agrees with the all-order QED result [12].1]

Thus we have performed the first systematic analysis of the high-energy asymptotic behaviour of the QCD amplitudes beyond the leading-power approximation and derived all-order double-logarithmic result for the leading mass-suppressed terms in typical two-scale problems. After separating the standard Sudakov factors the remaining non-Sudakov double-logarithmic corrections are described by two universal functions $g( \pm z)$ and $f( \pm z)$, Eqs. (44) and (10), of the variable $z=$ $\frac{\alpha_{s}}{4 \pi}\left(C_{A}-C_{F}\right) \ln ^{2}\left(m_{q}^{2} / Q^{2}\right)$ for the processes with single and double soft quark exchange, respectively. Note that in general the amplitudes with larger number of scattering particles, such as Bhabha scattering in QED [14], get contributions from both single and double soft fermion exchange and the corresponding asymptotic expressions involve both functions $g(z)$ and $f(z)$. These functions play the role of "Sudakov exponent" for the non-Sudakov double-logarithmic corrections. They are exponentially enhanced for large positive values of the argument and power suppressed for the large negative values. Our analysis reveals a nontrivial relation between the asymptotic behavior of different amplitudes and the amplitudes in different gauge theories. In particular, it demonstrates that if a QCD amplitude gets the exponential enhancement at high energy, the same amplitude in QED is suppressed by a power of the large logarithm, and vice versa. Note that the enhancement of the mass-suppressed amplitudes by the double-logarithmic corrections has been already observed in QED [14, 19]. The method introduced above is quite general and can be used for the

\footnotetext{
${ }^{1}$ In Ref. [12] only the singular part of the Sudakov corrections Eq. (2) has been factored out while $e^{-x}$ part has been absorbed into the definition of $F_{1}^{(1)}$. Such a decomposition however is not physical since the soft real emission cancels all the double logarithmic terms in Eq. (2) rather than its singular part.
} 
analysis of the more complex processes and observables such as Higgs boson production in association with a jet and the Higgs boson transverse momentum distribution, which however is beyond the scope of this Letter.

A.P. would like to thank Kirill Melnikov for numerous discussions and Babis Anastasiou for useful communication. The work of A.P. is supported in part by NSERC and Perimeter Institute for Theoretical Physics. The work of T.L. is supported by NSERC.

* ltao@ualberta.ca

† penin@ualberta.ca

[1] V. V. Sudakov, Sov. Phys. JETP 3, 65 (1956) [Zh. Eksp. Teor. Fiz. 30, 87 (1956)].

[2] J. Frenkel and J. C. Taylor, Nucl. Phys. B 116, 185 (1976).

[3] A. H. Mueller, Phys. Rev. D 20, 2037 (1979).

[4] J. C. Collins, Phys. Rev. D 22, 1478 (1980).

[5] A. Sen, Phys. Rev. D 24, 3281 (1981).

[6] G. F. Sterman, Nucl. Phys. B 281, 310 (1987).

[7] A. Ferroglia, M. Neubert, B. D. Pecjak and L. L. Yang, Phys. Rev. Lett. 103 (2009) 201601.

[8] A. Banfi, P. F. Monni and G. Zanderighi, JHEP 1401, 097 (2014).

[9] T. Becher and G. Bell, Phys. Rev. Lett. 112, 182002 (2014).

[10] D. de Florian, J. Mazzitelli, S. Moch and A. Vogt, JHEP 1410, 176 (2014).

[11] C. Anastasiou, C. Duhr, F. Dulat, E. Furlan, T. Gehrmann, F. Herzog and B. Mistlberger, JHEP 1503, 091 (2015)

[12] A. A. Penin, Phys. Lett. B 745, 69 (2015), Erratum: [Phys. Lett. B 771, 633 (2017)].
[13] K. Melnikov and A. Penin, JHEP 1605, 172 (2016).

[14] A. A. Penin and N. Zerf, Phys. Lett. B 760, 816 (2016), Erratum: [Phys. Lett. B 771, 637 (2017)].

[15] D. Bonocore, E. Laenen, L. Magnea, L. Vernazza and C. D. White, JHEP 1612, 121 (2016).

[16] R. Boughezal, X. Liu and F. Petriello, JHEP 1703, 160 (2017).

[17] I. Moult, I. W. Stewart and G. Vita, JHEP 1707, 067 (2017).

[18] T. Liu, A. A. Penin and N. Zerf, Phys. Lett. B 771, 492 (2017).

[19] V. G. Gorshkov, V. N. Gribov, L. N. Lipatov and G. V. Frolov, Sov. J. Nucl. Phys. 6, 95 (1968) [Yad. Fiz. 6, 129 (1967)].

[20] M. I. Kotsky and O. I. Yakovlev, Phys. Lett. B 418, 335 (1998).

[21] D. R. Yennie, S. C. Frautschi and H. Suura, Annals Phys. 13, 379 (1961).

[22] M. Beneke and V. A. Smirnov, Nucl. Phys. B 522, 321 (1998).

[23] V. A. Smirnov, Phys. Lett. B 404, 101 (1997).

[24] V. A. Smirnov, Applied asymptotic expansions in momenta and masses, Springer Tracts Mod. Phys. 177 (2002) 1.

[25] J. Frenkel and J. C. Taylor, Nucl. Phys. B 246, 231 (1984).

[26] C. Anastasiou, S. Beerli, S. Bucherer, A. Daleo and Z. Kunszt, JHEP 0701 (2007) 082.

[27] W. Bernreuther, R. Bonciani, T. Gehrmann, R. Heinesch, T. Leineweber, P. Mastrolia and E. Remiddi, Nucl. Phys. B 706, 245 (2005).

[28] W. Bernreuther, R. Bonciani, T. Gehrmann, R. Heinesch, T. Leineweber, P. Mastrolia and E. Remiddi, Nucl. Phys. B 712, 229 (2005).

[29] W. Bernreuther, R. Bonciani, T. Gehrmann, R. Heinesch, P. Mastrolia and E. Remiddi, Phys. Rev. D 72, 096002 (2005). 